\title{
On the Limit of some Diffusion-Reaction System with Small Parameter
}

\author{
H. GaJewski and H.-D. Sparneg
}

Es wird ein Reaktions-Diffusionssystem mit kleinem Parameter $\varepsilon$ betrachtet, das einen Polykondensationsproze $B$ beschreibt, in dem die chemische Reaktion schneller als der Massentransport verläuft. Für $\varepsilon \rightarrow 0$ ergibt sich eịe nichtlineare Evolutionsgleichung vom Typ $v_{t}=\Delta f(v)$.

Рассматривается система с малым параметром $\varepsilon$ с реакциями и диффузией и описывающая процесс поликонденсации, в котором химическая реакция протекает быстрее транспорта вешеств. Для $\varepsilon \rightarrow 0$ получается нелинеиное әволюционное уравнение типа $v_{t}{ }^{\circ}=\Delta f(v)$.

A diffusion-reaction system with small parameter $\varepsilon$ is considered describing some process of polycondensation in which the chemical reactions are faster than the mass transport. For $\varepsilon \rightarrow 0$ results a nonlinear evolution equation like $v_{t}=\Delta f(v)$.

Let $G \subset \mathbf{R}^{n}$. be a bounded domain, $\partial G=D=\bigcup_{1}^{r} D_{k}$ its smooth boundary with the components $D_{k}: D_{k} \cap D_{l}=\emptyset(k \neq l)$. In this note we want to study the following diffusion-reaction system for $x \in Q, t \in(0, T]=S, \varepsilon>0$ :

$$
\begin{aligned}
& \varepsilon A u=f(v)-u, \quad B u=0 \text { on } D \times S, \\
& \varepsilon v_{t}=u-f(v), \quad v(x, 0)=v_{0}=\text { const } \geqq 0 . \text { on } G
\end{aligned}
$$

whère $A$ is an elliptic differential operator with a suitable boundary operator $B$.

A problem of this kind occured when we tried to reduce some model of a polycondensation process in the so-called transport-limited case (see [7: eq. (5.3)]). The main question in that case was the convergence of the spatial $L^{1}$-norm of $v$ at each time $t$ because that implied the convergence of some other measurable quantity the average degree of polymerization.

Now we will show that under certain assumptions the solutions of (1) converge with $\varepsilon \rightarrow 0$ to the solution of

$$
v_{t}+A f(v)=\dot{0}, \quad v(x, 0)=v_{0}, \quad u=f(v)
$$

in $L^{2}(G \times S)$. A corollary will answer the question mentioned above. But (1) may be interesting even from a broader point of view. The second equation can be transformed to

$$
v_{t}+A_{\varepsilon} f(v):=v_{t}+A(I+\varepsilon A)^{-1} f(v)=0
$$

Here, $A_{\varepsilon}$ is of course the Yosida approximation of $A$. Thus, our convergence problem is a special case of the general question of the convergence of this approximation with inclosed nonlinearity. 
Investigations of a similar kind in the case of accretive operators in $L^{1}$ can be found in [2] and [3]. Other interesting diffusion-reaction equations with small parameters, even with mixed concentration terms on the right side of the equations, and their relationship to some nonlinear limit equation were studied by L. C. Evans in [4]. •

We assume the following conditions to be satisfied:

(F) $f: \mathbf{R} \rightarrow \mathbf{R}$ is an increasing function of class $C^{1}, f^{\prime}$ is locally Lipschitz continuous, $f(a)=a$, for some $a \leqq 0$.

(A) $A u=-\sum_{i=1}^{n} \frac{\dot{\partial}}{\partial x_{i}}\left(\sum_{j=1}^{n} a_{i j} u_{x_{j}}+a_{i} u\right)+a_{0} u$ on, $G$,

$B \dot{u}=\dot{\delta_{k}} \sum_{i=1}^{n}\left(\sum_{j=1}^{n} a_{i j} u_{x_{j}}+a_{i} u\right) \nu_{i}+b_{0} \dot{u}$ on $\quad D_{k}, \quad 1 \leqq k \leqq r$,

$a_{i j}\left(=a_{j i}\right), \quad a_{i} \in \dot{C}^{1+\mu}(\bar{G}), \quad 1 \leqq i, \quad j \leqq n, \quad a_{0} \in C^{\mu}(\bar{G}), \quad b_{u} \in C^{1+\mu}(D)$

there exists a $x>0$ such that $\sum_{i, j=1}^{n} a_{i j}(x) \xi_{i} \xi_{j} \geqq x|\xi|^{2}$ for all $x \in \ddot{G}$ and $\xi \in \mathbf{R}^{n}$; $a_{0} \geqq 0 ; \quad \operatorname{div} \vec{a}=0, \quad \delta_{k} \vec{a} \cdot \vec{n}+2 b_{0}^{\prime} \geqq 0, \quad 1 \leqq k \leqq r$, where $\vec{a}=\left(a_{1}, \ldots, a_{n}\right)$, $\vec{n}=\left(v_{1}, \ldots, v_{n}\right)-$ exterior normal to $D$

$\delta_{k} \in\{0,1\}, \delta_{k}=0$ implies $b_{0}=1$ on $D_{k} ; b_{0} \neq 0$.

Here and in the following an index $x$ or $t$ to some function will mean the partial 'derivative of this function with respect to the named 'variable.'

The notations $C^{l}, C^{l+a}, H^{l}$ describe the usual spaces (see e.g. [8]). $C^{a, b}(G \times H) \ni u$ means $u(\cdot, y) \in C^{a}(G), u(x, \cdot) \epsilon \cdot C^{b}(H)(x \in G, y \in H)$. By $\langle\cdot, \cdot\rangle$ we will always describe a pure $L^{2}$ - scalar product, not distinguishing - if there can be no misunderstanding - between the scalar products in $L^{2}(G), L^{2}\left(S, L^{2}(G)\right)$ or similar spaces.

Let us denote

$$
D^{\prime}=\breve{\cup}_{1}^{r}\left\{D_{k}: \delta_{k} \neq 0\right\}, \quad H=\left\{u \in H^{1}(G): B u=0 \text { on } D \backslash D^{\prime}\right\}
$$

Now we can identify the pair of operators $(A, B)$ with the operator $A: H \rightarrow H^{*}$ : given by

$$
\langle A u, v\rangle=\int_{G} \sum_{i, j=1}^{n} a_{i j} u_{x_{j}} v_{x_{i}} d x+\int_{G} a_{0} u v d x+\int_{G} \sum_{i=1}^{n} a_{i} u v_{x_{i}} d x+\int_{D^{\circ}} b_{0} u v d x
$$

It is known that $A$ has a continuous and isotone inverse (see e.g. $[1,8,5]$ ). The same is true for the "symmetric part" $A_{s}$ given by

$$
\left\langle A_{s} u, v\right\rangle=\int_{G i, j=1} \sum_{i}^{n} a_{i j} u_{x}, v_{x_{i}} d x+\int_{G} a_{0} u v d x
$$

Both, $A$ and $A_{s}$ are continuous operators themselves and strongly monotone. For $A_{s}$ this is quiet clear, in the case of $A$ it follows from the monotonicity of the "rest operator" $A_{r}=A-A_{s}$ :

$$
\begin{aligned}
\left\langle A_{r} u, u\right\rangle & =\frac{1}{2} \int_{G} \vec{a} \cdot \operatorname{grad}\left(u^{2}\right) d x+\int_{D^{\prime}} b_{0} u^{2} d x \\
& =\frac{1}{2} \int_{D^{\prime}} u^{2}\left(\vec{a} \cdot \vec{n}+2 b_{0}\right) d x \geqq 0
\end{aligned}
$$


Moreover, $A_{s}$ defines some new scalar products and equivalent norms in $H$ and $H^{*}$ :

$$
(u, v)_{H}=\left\langle A_{s}^{\prime} u, v\right\rangle, \quad(g, h)_{H^{*}}=\left\langle A_{s}^{-1} g, h\right\rangle .
$$

We, will use these new norms throughout this paper and note that thus $A_{s}$ becones the duality mapping between $H$ and $H^{*}$. Further, we should remark that $A_{r}: L^{2}(G) \rightarrow H^{*}$ is a continuous operator.

To prove convergence of the solutions to (1) we have first to make sure that there is anything to speak about at all. The solvability of (1) was already stated in [7] in a somewhat less general form. Nevertheless, the idea of the proof remains unchanged:

Thẹor̀m 1: Problem (1) has for fixed $\varepsilon>0$ exactly one solution

$$
u \in C^{2+\mu, \mu / 2}(\bar{G} \times \bar{S}), \quad v \in C^{\mu, 1+\mu / 2}(\bar{G} \times \bar{S})
$$

and it holds $a \leqq u \leqq f\left(v_{0}\right), a \leqq v \leqq v_{0}$.

Proof: (1) is equivalent to

$$
\begin{aligned}
& u=(I+\varepsilon A)^{-1} f(v), \\
& v_{t}^{\prime}=-\frac{1}{\varepsilon}\left(I-(I+\varepsilon A)^{-1}\right) f(v), \quad v(0)=v_{0} .
\end{aligned}
$$

$(I+\varepsilon A)^{-1}$ is continuous in $C^{\mu, 1+\mu / 2}(\bar{G} \times \bar{S})$ (see $\left.[1,8,5]\right)$, the same is true for $f$ because of $(F)$. So there exists a unique solution of the ordinary differential equation in $v$, local in time. To prove the global existence of this solution it suffices to show the maintained inequalities. The iterations

$$
\begin{aligned}
& u_{j}=(I+\varepsilon A)^{-1} f\left(v_{j-1}\right), \\
& v_{j \ell}=u_{j}-f\left(v_{j}\right), . v_{j}(0) \doteq v_{0} \quad(j \geqq 1)
\end{aligned}
$$

together with the maximum principle and the monotonicity of $f$ imply the monotonicity of the sequences

$$
\begin{aligned}
& a \leqq \cdots \leqq u_{j+1} \leqq u_{j} \leqq \cdots \leqq f\left(v_{0}\right), \\
& a^{\prime}=\cdots=v_{j+1}=v_{j}=\cdots=v_{0} .
\end{aligned}
$$

(To prove the validity of the bounds it suffices to note that $A f\left(v_{0}\right) \geqq 0$ and $A a \leqq 0$, $a=f(a)$ and $a \leqq v_{0}$.) Dini's Theorem and the uniqueness of the solution now guarantee the convergence of these iterations to the solution of (1) and thus the asserted inequalities

Corollary: The solutions $u, v$ of (1) belong to $C^{2,1}(G \times S)$.

This fact follows from the regularity assertion of Theorem 1 and the theorem about solutions of ordinary differential equations depending on parameters

The following theorem will be the main result of this paper. It gives an answer to the question what happens to (1) if $\varepsilon \rightarrow 0$. To distinguish between solutions for different parameters we will keep $\varepsilon$ as an index to these solutions. Further, set $Q=\dot{G} \times \dot{S}$.

Theorem 2: For $\varepsilon \rightarrow 0$ the solutions $\left(u_{\varepsilon}, v_{\varepsilon}\right)$ of (1) converge in the following sense:

$$
u_{s} \rightarrow f(v), \quad v_{\varepsilon} \rightarrow v \quad \text { in } L^{2}(Q),
$$


where $v$ is a function satisfying the following conditions

(i) $\quad v \in L^{\infty}(Q), \quad a \leqq v \leqq v_{0}$,

(ii) $, v \in H^{1}\left(S, H^{*}\right), \quad f(v) \in L^{2}(S, H)$,

(iii) $v$ is the unique solution of

$v_{t}+A f(v)=0, \quad v(x, 0)=v_{0} \quad$ a.e. in $G$

with the properties (i), (ii) mentioned above.

Proof: The proof of this theorem will be given in steps $(a)-(t)$.

(a) Theorem 1 and its corollary say that for every $\varepsilon>0$ we have $u_{\varepsilon}, v_{\varepsilon} \in C^{2,1}(Q)$, $a \leqq u_{\varepsilon} \leqq f\left(v_{0}\right), a \leqq v_{\varepsilon} \leqq v_{0}$ in $Q$.

(b) For every $\varepsilon>0$ it holds $f\left(v_{\varepsilon}\right) \geqq u_{\varepsilon}$ :

Put $w=u_{\varepsilon t}, z=v_{\varepsilon t}$. (1) implies

$$
\begin{aligned}
& A w=f^{\prime}\left(v_{\varepsilon}\right) z-w, \\
& z_{t}=w-f^{\prime}\left(v_{\varepsilon}\right) z, \quad z(0)=\frac{1}{\varepsilon}\left(u_{\varepsilon}(0)-f\left(v_{0}\right)\right) \leqq 0 .
\end{aligned}
$$

This problem has (in the sense of "=" replaced by " $\geqq$ " in every equation) the supersolution $W=Z=0$. For the iterated system

$$
\begin{aligned}
& A w_{n}=f^{\prime}\left(v_{\varepsilon}^{\prime}\right) z_{n-1}-w_{n}, \\
& z_{n t}=w_{n}-f^{\prime}\left(v_{\varepsilon}\right) z_{n}^{\circ}, \quad z_{n}(0)=z(0), \quad z_{0}=0 \quad(n \geqq 1)
\end{aligned}
$$

one shows with the usual mechanism $\left(R_{\varepsilon}:=(I+\varepsilon A)^{-1}\right.$ is an isotone operator!) that

$$
\begin{aligned}
& 0=W=w_{1} \geqq \cdots \geqq w_{n} \geqq w_{n+1} \geqq \cdots, \\
& 0=Z=z_{0} \geqq \cdots \geqq z_{n} \geqq z_{n+1} \geqq \cdots .
\end{aligned}
$$

On the other hand, we have

$$
w_{n}=R_{c}\left(f^{\prime}\left(v_{\varepsilon}\right) z_{n-1}\right), \quad z_{n}=F\left(z_{n-1}\right),
$$

where $F$ is a continuous homomorphism on $C(G)$ given by

$$
F(y)=\mathrm{e}^{-\frac{1}{3} \int_{0}^{t} f^{\prime}\left(v_{\varepsilon}\right) d s}\left(\frac{1}{\varepsilon} \int_{0}^{t} \mathrm{e}^{\frac{1}{\varepsilon} \int_{0}^{r} f^{\prime}\left(v_{\varepsilon}\right) d s} R_{\varepsilon}\left(f^{\prime}\left(v_{\varepsilon}\right) y\right) d r-z(0)\right)
$$

We want to prove that $F$ is a contraction in $C(G)$ for some norm equivalent to the original. This would give us the convergence of $\left(w_{n}, z_{n}\right)$ to the solution of (4) and thus the inequality $z \leqq 0$, which in connection with (1) and the definition of $z$ proves our statement.

Because of (a), (F) and the Lipschitz continuity of $R_{\varepsilon}$ we have

$$
\|F(b)-F(d)\|_{c} \leqq \frac{c}{\varepsilon} \mathrm{e}^{-\frac{1}{\varepsilon} \int_{0}^{t} f^{\prime}\left(v_{\varepsilon}\right) d s} \int_{0}^{t} \mathrm{e}^{\frac{1}{\varepsilon} \int_{0}^{r} f^{\prime}\left(v_{\varepsilon}\right) d s}\|b-d\|_{c} d r .
$$

Introducing the Bielecki norm

$$
\|y\|_{\gamma}=\sup \left\{\mathrm{e}^{-\dot{\gamma} t}\|y(t)\|_{c}: t \in S\right\}
$$

we get $\|F(b)-F(d)\|_{\gamma} \leqq \tilde{c}\|b-d\|_{\gamma}, \tilde{c}<1$ if $\gamma>\frac{c}{\varepsilon}$. 
(c) $u_{\varepsilon} \rightarrow u, v_{\varepsilon} \rightarrow v, f\left(v_{c}\right) \rightarrow z$ at least for some subsequences in the reflexive Banach space $L^{2}(Q)$ because of (a). (Here and in the following we will replace the whole (generalized) sequences by their converging parts not changing our notations.)

(d) : $\left\|f\left(v_{\varepsilon}\right)-u_{\varepsilon}\right\|_{L^{1}(\theta)} \rightarrow 0$ :

By (a), (b) and the second equation of (1) this sequence is bounded by $\varepsilon \cdot$ const and this expression tends to zero if $\varepsilon \rightarrow 0$.

(e) $\left\|f\left(v_{\varepsilon}\right)-u_{\varepsilon}\right\|_{L^{\prime}(Q)} \rightarrow 0$ :

This is true because $f\left(v_{\varepsilon}\right)$ and $u_{\varepsilon}$ belong to $L^{\infty}(Q)((a))$ and (d) holds.

(f) From the above statements we can now deduce $u=z$ a.e. in $Q$.

(g) $\left\{u_{e}\right\}$ is bounded in $L^{2}(S, H)$ :

Comparing the two equations of (1) we get $A u_{\varepsilon}=-v_{\iota t} \geqq 0((\mathrm{~b}))$ and because of $(\mathrm{a})$ $\left\{A u_{\varepsilon}\right\}$ is bounded in $L^{\mathbf{1}}(Q)$. Thus,

$$
c_{1} \geqq\left\|A u_{\varepsilon}\right\|_{L^{1}(Q)}\left\|u_{\varepsilon}\right\|_{L^{\infty}(Q)} \geqq\left\langle A u_{\varepsilon}, u_{\varepsilon}\right\rangle \geqq c_{2}\left\|u_{\varepsilon}\right\|_{L^{2}(S, H)}^{2} .
$$

Here we made use of the monotonicity of $A$ in the new norm.

(h) $u_{\varepsilon} \rightarrow u$ in $L^{2}(S, H)$ at least for some subsequence:

Because of $(\mathrm{g})\left\{u_{t}\right\}$ contains a weakly converging subsequence in this space. Because of (c) the limit of this subsequence must be $u$.

(j) $v_{e t} \rightarrow v_{t}$ in $L^{2}\left(S, H^{*}\right)$ at least for some subsequence:

$\left\{u_{\varepsilon}\right\}$ is bounded in $L^{2}(S, H)$ and $A$ is continuous. So $v_{e t}=-A u_{\varepsilon}$ is bounded in $L^{2}\left(S, H^{*}\right)$. Now we can repeat the argument used in (h) and recall the definition of weak derivatives.

(k) $\left\{u_{\varepsilon t}\right\}$ is bounded in $L^{2}\left(S, H^{*}\right)$ :

As before we have for $w_{\varepsilon}=u_{\varepsilon \ell}: A w_{\varepsilon}+w_{\varepsilon}=f^{\prime}\left(v_{\varepsilon}\right) v_{\varepsilon t}=: f_{\varepsilon}$. At first, we will show that $\left\{f_{c}\right\}$ is bounded in $L^{2}\left(S, H^{*}\right)$. It follows from (j) that $\left\{v_{\varepsilon l}\right\}$ is bounded in this space. For an arbitrary $\varphi \in L^{2}(S, H)$ we have $|\varphi| \in L^{2}(S, H)$ and because of (b)

$$
\left\langle f_{\varepsilon}, \varphi\right\rangle=\left\langle f^{\prime}\left(v_{\varepsilon}\right) v_{\varepsilon t}, \varphi\right\rangle \leqq \sup _{Q}\left(f^{\prime}\left(v_{\varepsilon}\right)\right)\left\|v_{\varepsilon t}\right\|_{L^{\varepsilon}\left(S, H^{*}\right)}\|\varphi\|\left\|_{L^{2}(S, H)} \leqq c_{3}\right\| \varphi \|_{L^{*}(S, H)}
$$

Herè we used once more condition (F). Now we have

$$
\varepsilon\left\|w_{\varepsilon}\right\|_{L^{z}(S, H)}^{2} \leqq c_{\ell}\left\|f_{\epsilon}\right\|_{L^{z}\left(S, H^{*}\right)}\left\|w_{\varepsilon}\right\|_{L^{z}(S, H)}
$$

because of the strong monotonicity of $A$. The continuity of $A$ on the other hand gives

$$
\left\|w_{\ell}\right\|_{L^{3}\left(S, H^{*}\right)} \leqq \varepsilon\left\|A w_{\varepsilon}\right\|_{L^{2}\left(S, H^{*}\right)}+\left\|f_{\varepsilon}\right\|_{L^{3}\left(S, H^{*}\right)} \leqq \varepsilon c_{5}\left\|w_{\varepsilon}\right\|_{L^{3}(S, H)}+\left\|f_{\ell}\right\|_{L^{2}\left(S, H^{*}\right)}
$$

and these two estimates taken together give the claimed result.

(l) From (g) and (k) it follows that $\left\{u_{e}\right\}$ is compact in $L^{2}(Q)$ (see e.g. [9: Theorem $5.1])$.

(m) This means the convergence of $u_{\varepsilon} \rightarrow u, f\left(v_{\varepsilon}\right) \rightarrow u$ in $L^{2}(Q)$ (for some subsequences).

(n) $u, v \in L^{\infty}(Q), a \leqq u \leqq f\left(v_{0}\right), a \leqq v \leqq v_{0}$ a.e. in $Q$ :

We know already that $u$ and $v$ are weak limits in $L^{2}(Q)$ of some sequences which satisfy the given inequalities. Then there exist some subsequences and some convex combinations of them which converge in the strong sense (see e.g. [10: Theorem I, 1.1.8]), but this implies the convergence of some new subsequences a.e. in $Q$. Of course, $u$ and $v$ as their limits will satisfy the same inequalities.

(o) $u=f(v)$ a.e. in $Q$ :

$x=\dot{v}-\xi(f(v)-u) \in L^{\infty}(Q)(\xi>0)$ and because of the monotonicity of $f((\mathrm{~F}))$ we have

$$
\xi\langle u-f(x), f(v)-u\rangle=\lim _{\varepsilon \rightarrow 0}\left\langle f\left(v_{\varepsilon}\right)-f(x), v_{\varepsilon}-x\right\rangle \geqq 0 .
$$


If $\xi \rightarrow 0$, then this can be true only for $u=f(v)$.

(p) $v_{\varepsilon} \rightarrow v=f^{-1}(u)$ in $L^{2}(Q)$ at least for some subsequence:

Because of (F) there exists $f^{-1}$ and is a continuous function. Our hypothesis now follows from $(\mathrm{m})$ and $(\mathrm{o})$ by Lebesgue's Theorem.

(q) $v_{t}+A f(v)=0$ (as an equation in $L^{2}\left(S, H^{*}\right)$ ):

The statements (j) and $(\mathrm{h})$ together with (o) ensure that the term on the left is well defined. Further, for an arbitrary $\varphi \in L^{2}(S, H)$ we have

$$
\left\langle v_{t}+A f(v), \varphi\right\rangle=\lim _{\varepsilon \rightarrow 0}\left\langle v_{\varepsilon t}{ }^{\prime}+A u_{\varepsilon}, \varphi\right\rangle=0
$$

(r) $v(0, x)=v_{0}$ a.e. in $G:$

The proof of this fact amounts to a repetition of the arguments of $(\mathrm{c})$ and $(\mathrm{n})$ applied to $v(0, \cdot)$ instead of $v$ :

(s) Uniqueness of the solution:

Let $v$ 'and $w$ be two solutions of the equation given in (q) which satisfy (i) and (ii), set $z=v-w$. For $t \in S$ put $S_{t}=(0, t], Q_{t}=G \times S_{\ell}$. Because of (F) we have

$$
\langle f(v)-f(w), z\rangle_{L^{2}\left(Q_{t}\right)} \geqq M\|f(v)-f(w)\|_{L^{\mathbf{z}}\left(Q_{t}\right)}^{2}
$$

where $1 / M$ is the Lipschitz constant of $f$ in the interval $\left[a, v_{0}\right]$. The continuity of $A_{r}: L^{2} \rightarrow H^{*}$ on the other hand gives

$$
\left\|A_{r}(f(v)-f(w))\right\|_{L^{2}\left(S_{t}, H^{*}\right)} \leqq c_{B}\|f(v)-f(w)\|_{L^{2}\left(Q_{t}\right)} .
$$

Thus, we get

$$
\begin{aligned}
& 0=\left(z_{t}+A(f(v)-f(w)), z\right)_{L^{2}\left(S_{t}, H^{*}\right)} \\
& =\frac{1}{2}\|z(\ell)\|_{H^{\bullet}}^{2}+\left\langle A_{s}^{-1}\left(A_{s}+A_{r}\right)(f(i)-f(w)), z\right\rangle \\
& =\frac{1}{2}\|z(t)\|_{H^{*}}^{2}+\langle f(v)-f(w), z\rangle+\left(A_{r}(f(v)-f(w)), z\right)_{L^{2}\left(S_{\ell} H^{*}\right)}
\end{aligned}
$$

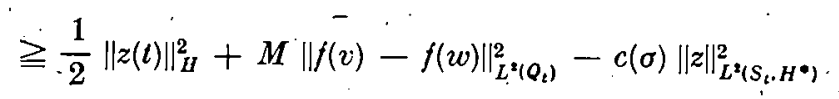

$$
\begin{aligned}
& -\sigma c_{6}{ }^{2}\|f(v)-f(w)\|_{L^{2}\left(Q_{t}\right)}^{2}
\end{aligned}
$$

We can now choose $\sigma$ sufficiently small to get $M \geqq \sigma c_{6}^{2}$. This implies

$$
2 c(\sigma) \int_{0}^{t}\|z(r)\|_{H^{*}}^{2} d r \geqq\|z(t)\|_{H^{*}}^{2},
$$

a situation which by Gronwall's Lemma leads to $z=0$. This ends the proof of the uniqueness statement.

(t) The uniqueness of the solution now guarantees a posteriori the convergence of all those sequences for which we had upto now only shown the convergence of , certain subșequences. This completes the proof of Theorem 2

Corollary: The convergence $v_{\varepsilon}(t) \rightarrow v(t)$ in $L^{2}(G)$ is true for all $t$ of $S$.

Proof: By (c) and (j) in the proof of the preceding theorem it holds $v_{\varepsilon} \rightarrow v$ in $H^{1}\left(S, H^{*}\right) \subset C\left(S, H^{*}\right)$. Moreover, $\left\|v(t)-v_{\varepsilon}(t)\right\|_{L^{2}(G)} \leqq$ const $((\mathrm{a}),(\mathrm{n}))$. Now, for an arbitrary $h \in L^{2}(G)$ there exists a sequence $\left\{h_{n}\right\} \subset H, h_{n} \rightarrow h$ in $L^{2}(G)$ and for fixed 
$t \in S$ we have

$$
\begin{aligned}
\left\langle v_{\varepsilon}(t)-v(t), h\right\rangle= & \left\langle v_{\varepsilon}(t)-v(t), h-h_{n}\right\rangle+\left\langle v_{\varepsilon}(t)-v(t), h_{n}\right\rangle: \\
\cdot & \leqq c_{7}\left\|h-h_{n}\right\|_{L^{z}(G)}+\left\langle v_{\varepsilon}(t)-v(t), h_{n}\right\rangle .
\end{aligned}
$$

We can now choose $n$ to make the first expression on the right side smaller than a given bound and then choose $\dot{\varepsilon}$ to do the same with the second term

Remark 1: Because of (a) and ( $n$ ) the definition of $f$ is important only in the interval $\left[a, v_{0}\right]$. This is true in the case of Theorem 1, too. Thus, we can weaken condition $(\mathbf{F})$.

Remark 2: If $f$ is only a nondēcreasing function which satisfies the other conditions of $(F)$, then the proof of Theorem 2 remains valid with the exception of step (p). In that case we can only prove the weak convergence of $v_{\varepsilon}$ in $L^{2}(Q)$.

\section{REFERENCES}

[1] Amavs, H.: Invariant sets and existence theorems for semilinear parabolic and elliptic systems. J. Math. Anal. Appl. 65 (1978), 432-467.

[2] Crandall, M. G.: An introduction to evolution governed by accretive operators. In: Dynamical systems - an international symposium (Ed.: L. Cesari, J: Hale and J. LaSalle). New York 1977, pp. 131-165.

[3] Crandat.l, M.G., and M. Pierre: Regularization effects for $u_{t}+A \varphi(u)=0$ in $L^{1}$. MRC Technical summary report \# 21S7, Univ. Wisconsin 1981.

[4] Evans, L. C.: A convergence theorem for a chemical diffusion-reaction system. Houston J. Math. 6 (1980), 259.

[5] GAJEwSKI, H., and J. GÄRTNER: On the asymptotic behaviour of some reaction-diffusion processes. Math. Nachr. 102 (1981), $141-155$.

[6] GaJewski, H., Gröger, K., and K. Zacharias: Nichtlineáre Operatorgleichungen und Operatordifferentialgleichungen. Akademie-Verlag: Berlin 1974.

[7] Gajewski, H., and H.-D. Sparing: On a model of a polycondensation process with thermal degradation. ZAMMI 62 (1982), 616-626.

[8] Ладыненская, О. А., И Н. Н. УРАЛЬцевА: Јинейные и квазилинейные уравнения эллиптического типа. Изд-во Наука: Москва 1964.

[9] Lions, J.-L.: Quelques méthodes de résolution des problémes aux limites non linéaires. Dunod: Paris 1969.

[10] TANABE, H.: Equations of evolution. London 1979.

Manuskripteingang: 01.08.1983.

\section{VERFASSER :}

Prof. Dr. H. GaJewski and Dr. H.-D. Sparing

Institut für Miathematik der Akademie der Wissenschaften der DDR

DDR-1080 Berlin, Mohrenstr. 39 